\title{
Cyber-Physical Systems for Water Sustainability: Challenges and Opportunities
}

\author{
Zhaohui Wang, Michigan Technological University \\ Houbing Song, West Virginia University \\ David W. Watkins, Keat Ghee Ong, and Pengfei Xue, Michigan Technological University \\ Qing Yang, Montana State University \\ Xianming Shi, Washington State University
}

\begin{abstract}
Water plays a vital role in the proper functioning of the Earth's ecosystems, and practically all human activities, such as agriculture, manufacturing, transportation, and energy production. The proliferation of industrial and agricultural activities in modern society, however, poses threats to water resources in the form of chemical, biological, and thermal pollution. On the other hand, tremendous advancement in science and technology offers valuable tools to address water sustainability challenges. Key technologies, including sensing technology, wireless communications and networking, hydrodynamic modeling, data analysis, and control, enable intelligently wireless networked water Cyber-Physical Systems (CPS) with embedded sensors, processors, and actuators that can sense and interact with the water environment. This article will provide an overview of water CPS for sustainability from four critical aspects: sensing and instrumentation, communications and networking, computing, and control, and explore opportunities and design challenges of relevant techniques.
\end{abstract}

\section{WATER SUSTAINABILITY}

Water is the lifeblood of the planet. Safe and abundant water resources are critical to all three dimensions of sustainability: social, economic, and environmental. Given its close linkage to a number of key global challenges, including population growth, industrialization, and climate change, water sustainability is of critical importance for sustainable development in the modern world. Achieving water sustainability, in turn, requires achieving universal access to safe drinking water, sanitation and hygiene; improving the efficiency of water use for economic purposes; strengthening equitable, participatory and accountable water governance; improving wastewater management and protecting water quality; and reducing the risks of natural and human-induced water-related disasters. In industrialized nations, fresh water resources - ground water, lakes, rivers, and streams - must meet a number of (sometimes competing) water use needs, including domestic, commercial, industrial, agricultural, and energy generation. In addition, sustainability requires that water withdrawals and consumption for human uses do not compromise aquatic ecosystems.

Although water is a renewable resource, water availability and water quality may not be sufficient for desired water uses and ecosystem functioning. Deficits, or scarcity, may occur at seasonal time scales and at geographic scales ranging from community water systems to regional river basins and groundwater aquifers. In addition to overuse or scarcity, water resources are facing many other severe challenges, including contamination, aging infrastructure, lack of data for informed decision making, weak public awareness of water challenges, and inefficient water management strategies. Many of these challenges are due to global change issues, such as population growth, economic development and climate change, which are expected to increase in the foreseeable future.

Given these critical and persistent water resources challenges, we advocate "green" ${ }^{1}$ " applications of the information and communications technology (ICT) to help achieve water sustainability [1]. To this end, green ICT can provide many benefits, including improved water quality, more effective emergency response, and increased public awareness of environmental challenges. We envision technologies including communications and networking, sensor technology, hydrodynamic modeling, data analysis, and human-centered decision support systems to enable intelligently networked water CyberPhysical Systems (CPS). This article will explore opportunities and design challenges of CPS for promoting water sustainablility, including sensing and instrumentation, communications and networking, computing, and control. Given the diversity of water sustainability challenges, techniques discussed in this article could be further tailored to specific application requirements.

\section{WATER Cyber-PhysicAl Systems}

Cyber-Physical Systems (CPS) are intelligently networked systems with embedded sensors, processors and actuators that are designed to sense and interact with the physical world (including human users), and support real-time, guaranteed performance in safety-critical applications, as defined in a CPS Vision Statement published in 2012 by the Federal Networking and Information Technology Research and Development (NITRD) Program's CPS Senior Steering Group. The interplay between the "cyber" and "physical" elements among the CPS is critical: sensing, networking, computing, and control need to be deeply integrated in every component of CPS, and the CPS components must be inter-operable with a concerted design. Just as the Internet transformed the way people interact with information, CPS is transforming the way people interact with engineered systems and promoting sustainability.

\footnotetext{
${ }^{1}$ Here "green" indicates the application of ICT to address environmental sustainability challenges.
} 


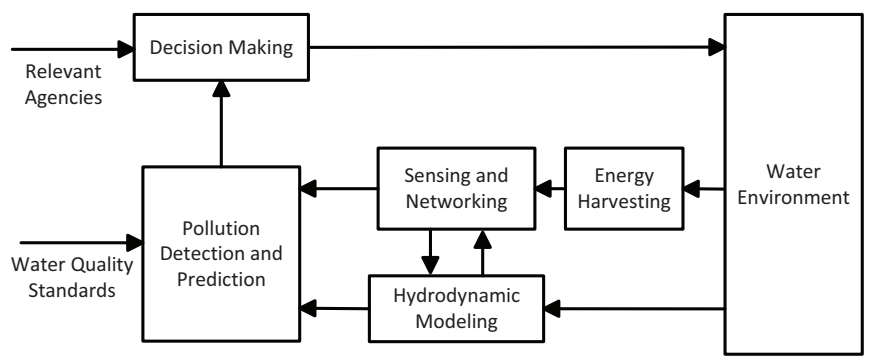

Fig. 1. Architecture of a typical water CPS. Arrows indicate the flow of data, computing results, and/or control commands.

\section{A. Water Cyber-Physical Systems (CPS)}

A typical water CPS architecture is depicted in Fig. 1. The water CPS monitors water quality conditions in real-time, and detects water pollution events quickly. Hydrodyamic modeling is integrated with the real-time measurements from various water quality sensors to generate the predicted transport of pollutants in the water environment, which is feedback into the input of the water CPS for optimal decision making of reactive and proactive actions to respond to water contamination emergencies effectively, thus forming a close-loop water CPS. In addition, energy harvesting is incorporated for a sustainable water CPS.

To develop a water CPS, we need (i) sensing, communications and networking technologies to enable flexible, reliable, and high performance distributed networking within a water CPS, that provide an accurate and reliable model of the water infrastructure and enable time-aware and timecritical functionalities, and (ii) computing technologies such as computational modeling, data management, machine learning, and other tools to understand, address, and communicate water sustainability challenges. In addition, (iii) adaptive and predictive hierarchical hybrid control technologies are critical to achieve tightly coordinated and synchronized actions and interactions in a water CPS that is intrinsically synchronous, distributed and noisy. Based on the CPS Vision Statement published by NITRD $^{2}$, Table I provides a comprehensive summary of key technologies for developing a water CPS.

\section{B. Example Applications of CPS for Water Sustainability}

The application of water CPS could increase the efficiency, reliability, security, and people's confidence in different types of water systems, hence setting the path towards water sustainability. In recent years there have been tremendous advances in real-time water quality sensing, communications and computing, which have great potential for ubiquitous environmental monitoring and interaction. Using these technologies, costeffective infrastructures can collect in-situ data remotely and on a continuous basis, as well as store, communicate, analyze, and visualize it in real time. However, compared to other applications of CPS, there has been relatively limited research on CPS for sustainable water management. In this section, we present two applications of CPS for promoting water sustainability.

\footnotetext{
${ }^{2}$ Source: http://www.nitrd.gov/nitrdgroups/images/6/6a/Cyber_Physical_ Systems_\%28CPS\%29_Vision_Statement.pdf
}

1) Water distribution system monitoring: Water distribution system monitoring is an important application of CPS in the water sector. In this application, the traditional real-time water quality monitoring performed at water treatment plants is extended to multiple locations within a water distribution system for contamination warning. The water CPS monitors baseline water quality conditions continuously in real-time such that a sudden change in water quality parameters can trigger a contamination warning. Benefits of this water CPS are improved water quality closer to the point-of-use, along with additional security for detecting intentional or unintentional contamination events within the system. A related CPS application is for control and mitigation of water losses in distribution systems (in the United States, there are an estimated 240,000 water main breaks per year). In this case, continuous monitoring of water pressure at various points in the system and automatic closure of valves can stop the flow of water to a broken section. Although the use of such on-line water distribution system monitoring and early warning systems is becoming common [2], water utilities are challenged by realtime data management and decision support, and there is a need for improved predictive models of contaminant transport [3].

2) Source water quality monitoring: Source water quality monitoring can be critical for advance warning and emergency management. The water sector is vulnerable to a wide range of chemical, biological, and radiological releases. In the United States, there are nearly 14,000 oil spills reported per year, and such emergencies can affect large populations. For example, on January 9, 2014, crude 4-methylcyclohexane methanol (MCHM), a chemical primarily used to clean coal, leaked from a storage tank near Charleston, West Virginia, and bled into a river upstream of a water-treatment plant. As a result, about 15 percent of the state's residents were advised not to drink the water.

Despite the need to safeguard water supplies and to protect public and ecosystem health, real-time monitoring of ambient environmental conditions is uncommon, primarily due to technological and cost limitations [4]. Some notable examples of ambient monitoring systems are a network of monitoring buoys on the Mississippi, Missouri and Illinois Rivers developed by the National Great Rivers Research and Education Center (NGRREC); and the Intelligent River project pursued at Clemson University. Example projects monitoring aquatic ecosystem health (e.g., nutrients, invasive species) include the Jefferson project at Lake George, N.Y.; the Hudson River Environmental Conditions Observing System (HRECOS); and the River and Estuary Observatory Network (REON).

With remote, in-situ, and real-time water monitoring and warning systems in place, computing and communications technologies can facilitate the distilling of information from potentially vast amounts of data, as well as timely dissemination to decision makers and the public. Furthermore, hydrodynamic simulation modeling can provide forecasts of the scope of the emergency and contribute to effective response. Computing technologies can help keep track of numerous details in all stages of an emergency (forecast, warning, and response), helping people grasp the dynamic of a disaster and 
TABLE I

KEy TECHNOLOGIES TO DEVELOP A WATER CPS (BASED ON THE CPS Vision STATEMENT By NITRD, 2012).

\begin{tabular}{|l|l|}
\hline Key Technologies & Role \\
\hline $\begin{array}{l}\text { Distributed sensing, communications and } \\
\text { perception }\end{array}$ & Enable time-aware and time-critical functionality \\
\hline Adaptive and predictive hierarchical hybrid control & $\begin{array}{l}\text { Achieve tightly coordinated and synchronized actions and interactions in water CPS that is } \\
\text { intrinsically synchronous, distributed and noisy }\end{array}$ \\
\hline Diagnostics and prognostics & Identify, predict, and prevent or recover from faults \\
\hline Autonomy and human interaction & Facilitate model-based design of reactive water CPS that is used by humans \\
\hline Validation, verification, and certification & Ensure high confidence in system safety and functionality \\
\hline Abstractions, modularity and composability & $\begin{array}{l}\text { Enable water CPS system elements to be combined and reused while retaining safety, security, } \\
\text { and reliability }\end{array}$ \\
\hline $\begin{array}{l}\text { Systems-engineering based architectures and } \\
\text { standards }\end{array}$ & $\begin{array}{l}\text { Enable efficient design and development of reliability systems while ensuring interoperability and } \\
\text { integration with legacy systems }\end{array}$ \\
\hline $\begin{array}{l}\text { Integration of multi-physics models and models of } \\
\text { software }\end{array}$ & $\begin{array}{l}\text { Enable co-design of physical engineered and computational elements with predictable system } \\
\text { behaviors }\end{array}$ \\
\hline Cyber-security & Guarantee safety by guarding against malicious attacks \\
\hline
\end{tabular}

make quicker and better decisions.

In the sequel, we will explore opportunities and design challenges of water CPS in four critical and complementary aspects, including (i) sensing and instrumentation, (ii) communications and networking, (iii) computing, and (iv) control.

\section{SEnsing AND InStRUMENTATION}

\section{A. Sensing and Instrumentation}

Advances in computer and electrical engineering in the past two decades have significantly reduced the size, cost, and power requirement of digital electronics. Low-cost, lowpower devices with sufficient storage and data processing capabilities have led to the proliferation of wireless sensing in a wide range of applications. However, real-time and in-situ measuring of water quality and quantity has been progressing slowly, primary due to the harsh environment sensors and instruments have to endure.

Fostering water sustainability requires continuous monitoring of multiple parameters such as dissolved oxygen, flow rate, turbidity, conductivity, $\mathrm{pH}$, and/or suspected chemical or biological pollutants. For an autonomous monitoring system, the sensors used in tracking these parameters need to be stable and accurate for a long period of time. Exposure to harsh weather and temperature changes can post a challenge for the long term durability of sensors, but proper sensor packaging and use of high-performance electronic components can mitigate this issue. Another challenge is the biofouling effect, where aquatic fauna and flora aggregate on or around the sensors, preventing them from operating normally. Biofouling is the major limitation to longevity for many underwater sensors.

Many techniques have been deployed to reduce or limit biofouling effects. Physical cleaning such as scraping or wiping the sensors at fixed time intervals is effective but labor intensive. A common unmanned antifouling strategy is the long term deployment of biocides around the sensors. The biocides are usually embedded into surfaces of the sensor housings or coating layers protecting the sensor housings to be slowly leached out [5]. In addition, electro-mechanical principles have been employed to reduce biofouling [6]. Mechanical vibrations have been shown to remove biofouling, but the associated power requirement is high. Direct electrification of organisms

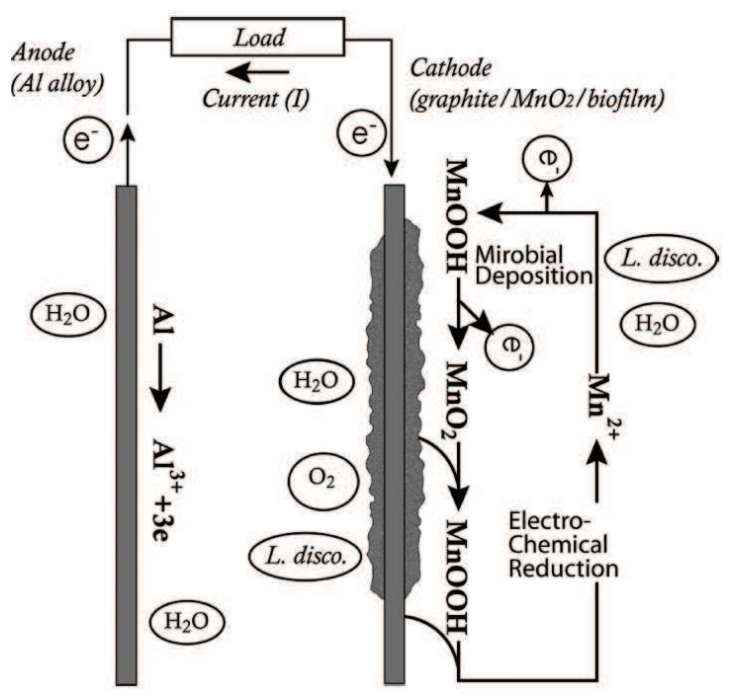

Fig. 2. Schematic of the novel MFC, consisting of a sacrificial anode of aluminum alloy and a cathode of porous graphite covered by manganese dioxide and L. discophora biofilms [7].

has also been tested to remove fouling organisms from the sensor surface.

Low-cost sensing and computing devices have been a major driving force of a widespread use of wireless sensing techniques in the terrestrial radio environment. However, the cost of water sensing devices - such as underwater vehicles and acoustic communication modems - could be several orders of magnitude higher than their terrestrial radio counterparts, which significantly hinders the research progress and the broad use of underwater wireless sensing techniques. Research towards cost-effective instrumentation is essential for expanded use of remote, in-situ, real-time, and continuous water monitoring.

\section{B. Energy Harvesting}

To achieve a water CPS, it is desirable to use an array of sensors to sense environmental parameters in a wide water area and employ wireless devices to transmit water quality information back to a data collection or processing center. 
However, batteries become the bottle neck of such a system as they need to be replaced, after being exhausted, in a relatively short period of time. Replacing batteries for large amount of sensors is time- and labor-consuming and likely to miss events that occur during replacement of batteries. Therefore, using energy harvested from water becomes the key component in achieving a sustainable water CPS.

Energy harvesting process could be used in a water CPS to convert energy from ambient sources in water to power a set of small wireless devices. However, traditional energy sources, e.g., solar power, thermal energy and kinetic energy, may not be easily accessed in a water environment. For example, solar power and thermal energy are very limited in deep ocean, and kinetic energy may not exist in a lake.

We advocate an alternative energy harvesting technique, which harvests biological energy via microbial fuel cells (MFCs) that generate electricity through electrochemical reactions with a type of common and safe bacteria (magnesium oxidizing microorganisms) ubiquitous in water [7]. As illustrated in Fig. 2, biocathode MFCs that harness the native population of magnesium oxidizing microorganisms (MOM) abundant in natural waters will be made self-sufficient simply with the pretreated electrodes and nutrients continuously available in the environment. They can deliver potential renewable power (voltage up to $1.0 \mathrm{~V}$ and current up to $1.2 \mathrm{~mA}$ ) in the aqueous water environment.

Due to the fluctuation of energy generated from MFCs, a power management system will be needed to provide a stable and accurate DC output to power sensors. Unlike conventional or commercially available power converters for low voltage applications, the desired power convertors need to provide a stable and accurate output to sensors even when the voltage of the MFCs fluctuates in a wide range or drops to a very low level such as below $0.5 \mathrm{~V}$. Innovative and efficient design is needed to realize such a power management system.

\section{WiRELESS COMMUNiCATIONS AND NeTWORKING}

\section{A. Wireless Heterogenous Networks}

Though direct access to the aquatic environment has been difficult, wireless communication technology makes unmanned water monitoring possible. Equipped with wireless (radio or acoustic) communication capabilities, sensing and actuation modules can be remotely controlled for specific tasks and transmit monitoring information to a centralized computer for analysis.

Especially about real-time monitoring of ambient aquatic conditions, the complexity of aquatic environment calls for intelligently networked systems which could consist of several types of sub-networks. Fig. 3 depicts a hybrid wireless networked system constituted by a surface buoy sub-network and an underwater sub-network with possibly mobile vehicles. The underwater sensing nodes can be anchored at the water bottom or float with water currents, and collect data samples at various water depths. Due to the large attenuation of electromagnetic waves in water, each underwater node has to rely on sound as the information carrier to communicate, hence is often equipped with an acoustic modem. The surface buoys could have different types of sensors for collecting near-surface data samples as well as meteorological information, and be equipped with (i) acoustic modems for communications with underwater nodes and (ii) radio-frequency (RF) modems for communications above water surface with other buoys and a control center.

Although data collection could be fulfilled by a surface buoy network through hanging sensors at different depths of the water column, incorporating an underwater acoustic sub-network yields several practical advantages. First, the underwater nodes do not disturb water surface activities (e.g., recreation and shipping). Secondly, they could maintain desired network functionalities in harsh weather conditions (e.g., stormy periods, and winter seasons with ice coverage). And lastly, they reduce the wireless network vulnerability to tempering or pilfering.

\section{B. Challenges}

Challenges in the above wireless heterogenous network design lie in a concerted network architecture and protocol suite under specific application requirements.

First, a majority of existing research on surface buoy networking adopts the Zigbee standard and operates in a clustered networking architecture. Data samples collected at each cluster head are sent to a remote monitoring center or the Internet via existing cellular/satellite networks. Despite intensive research in this area, long-range multi-hop data transmission along a river path or within a complex urban environment remains a challenging problem.

Secondly, compared to the terrestrial radio networking, underwater acoustic networking is still in its early stage, primarily because of the distinct features of underwater acoustic channels: (i) the underwater acoustic signal propagates at a speed $(\sim 1500 \mathrm{~m} / \mathrm{s})$ five orders of magnitude lower than the radio speed in air $\left(\sim 3 \times 10^{8} \mathrm{~m} / \mathrm{s}\right)$, leading to very large signal propagation latency; (ii) due to frequency-dependent sound attenuation, the underwater acoustic channel has a much lower bandwidth $(\sim \mathrm{kHz})$ than the surface RF channel $(\sim$ $\mathrm{MHz}$ ); and (iii) underwater acoustic links often suffer large temporal variations due to environment dynamics. Given the above differences between RF links and acoustic links, the RF connection among buoys can be regarded as a backbone for underwater acoustic networking, and multiple geographically distributed buoys could be deployed to reduce the end-to-end transmission latency and boost the end-to-end packet delivery performance. Leveraging the surface RF connection, many underwater networking protocols have been proposed, which however, lack sufficient experimental validation to demonstrate their performance in practical systems.

Thirdly, the optimal deployment of heterogeneous nodes is another challenge in a water CPS. Depending on application scenarios (e.g., the water depth), the sensing nodes could be regarded as deployed on a 2-dimensional plane (e.g., water surface or bottom) or in a 3-dimensional water column [8]. Existing research often takes communication coverage, sensing coverage, end-to-end transmission latency, network reliability, and network resilience to node failures as performance metrics, 


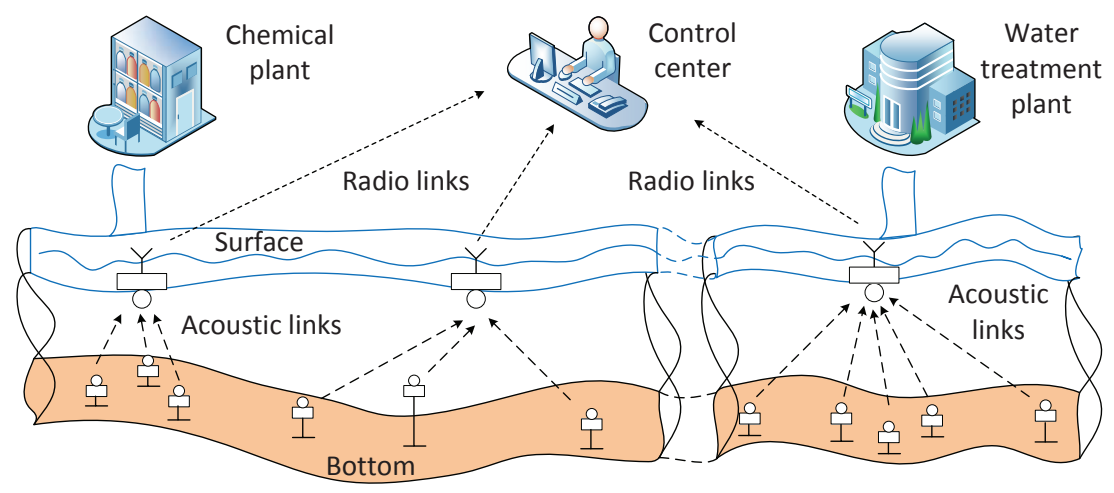

Fig. 3. An illustration of a 3-dimensional hybrid wireless network architecture.

and investigates the deployment of stationary networks with both surface buoys and underwater nodes, mobile underwater data collectors, or mobile surface buoys. Note that water parameters of interest often follow certain spatial-temporal distributions that can be simulated via hydrodynamic modeling. We anticipate more research on sensor node deployment that takes into account the spatial-temporal distribution of water parameters.

\section{Computing Technologies}

Computing technologies offer a wide range of tools and methodologies for understanding, addressing, and communicating water sustainability challenges. However, meeting these challenges will involve advances in a number of computing research fields. In this section we will examine the opportunities and challenges of computing technologies for water sustainability in two major areas.

\section{A. Hydrodynamic Modeling}

Although real-time and in-situ water sensing could provide continuous measurements of water parameters of interest at specific sites, understanding their spatial and temporal distributions relies on computational modeling. Hydrodynamic modeling can be brought to bear in several ways: to refine the grid size and time step of aquatic sensing, to predict future aquatic conditions, and to simulate multiple events or scenarios to improve decision making for water sustainability.

Although hydrodynamic modeling has seen rapid development in the past three decades, it remains a challenge to resolve hydrodynamic processes at multiple scales. Coastal marine and fresh water systems typically consist of inner shelves, estuaries and inland lakes, which are characterized by complex coastlines, series of islands and peninsulas, inlets, and extensive intertidal marshes. The irregular geometry has limited the ability of models to adequately resolve fine scale processes, which may be critical for predicting local conditions such as contamination of a water intake. Furthermore, the coupled dynamics among the hydrosphere, atmosphere and lithosphere complicate the driving factors of the hydrodynamic simulation at a wide range of different scales. Nonetheless, driven by computing technology advances, such as adaptive mesh refinement, code parallelization, and GPU-accelerated computing [9], [10], hydrodynamic modeling has entered a period unprecedented towards resolving complex biophysical processes at scales appropriate for operational forecasting: curvilinear and unstructured grid modeling can now resolve irregular geometries, and multi-grid approaches allow the resolution of small-scale features within a larger spatial domain [11].

\section{B. Data-Driven Decision Making}

In-situ aquatic sensing data and hydrodynamic modeling results can support decisions and subsequent actions to safeguard water sustainability. When appropriate, automatic decision making could be carried out through optimization algorithms; one example is automatic water pollution detection, warning, and response. Alternatively, decision support systems can help multiple parties - emergency managers, water utility companies, and policy makers and governments - distill information and coordinate with each other to make betterinformed decisions.

Challenges in data-driven decision making include the following:

- Heterogeneity of data sets: Data sets from different observation systems could bear very different meanings and structures, and represent a wide range of spatiotemporal scales. For instance, in the aquatic ecosystem monitoring, relevant data sets might include remotely sensed data from satellites, meteorological and oceanographic information from surface observation networks, and data from underwater acoustic sensor networks;

- Uncertainty of hydrodynamic models: Modeling realworld phenomena suffers from both aleatoric uncertainties (e.g., turbulent flows) and epistemic uncertainties (e.g., lack of physical measurements), which often lead to models performing worse with verification data sets than with calibration data sets. Though hydrodynamic modeling can be very accurate, many unknowns still exist, especially in complex hydraulic systems;

- Sequential decision making: By definition, sustainable water systems are designed to operate over long time horizons. Sequential decision making is required based on continuous data samples, continuously improved models and sensor networks, and improved outcomes of decisions over time. 
In the face of these challenges, machine learning and data mining techniques can be adopted for event detection and data-driven decision making. Machine learning techniques, including both supervised leaning (e.g., linear regression, neural networks, and decision trees) and unsupervised learning (e.g., clustering algorithms) have been developed for water quality event detection [12]. Data mining, data assimilation and uncertainty estimation methods can be used with hydrodynamic models in real-time response as well as to continuously improve model calibration over time [13]. Probabilistic forecasting methods, now becoming common in water management at seasonal timescales, can be adapted for sequential decision making during contamination events [14]. For improved emergency response planning, scenario analysis and agent-based modeling hold promise for helping decision-makers account for complexity and hedge against contingencies [15].

\section{Control Technologies}

Control technologies, particularly feedback control and realtime control, are needed to design a water CPS with desired behaviors for sustainability.

A CPS should have integrated protection, detection, and response mechanisms to be able to survive natural disasters, human error, and cyber attack without loss of function. This could be achieved with control technologies, which provide a systematic approach to designing feedback loops that are stable in that a CPS avoids wild oscillations, accurate in that a CPS achieves objectives such as target response times for service level management, and settle quickly to its steady state values.

Compared with feed-forward control, also called anticipative control, which is a control mechanism that predicts the effects of measured disturbances and takes corrective action to achieve the desired result, feedback control is a control mechanism that uses information from measurements to manipulate a variable to achieve the desired result and offers more advantages, including versatility and robustness. A water CPS featuring feedback loops, where physical processes affect computations and vice versa, is desirable. In a closed-loop CPS for real-time water quality monitoring and pollution detection, the water environment and hydrodynamic modeling affect the accuracy of pollutant transport prediction; the predicted pollutant transport affect the decision making about how to respond to water emergencies and the consequences of the water emergencies to the water environment.

A water CPS for sustainability should also be a real-time control system in which its temporal properties are essential for reliability and correctness. Depending on the consequences that may occur because of a missed deadline, a real-time control system can be distinguished in three categories: hard, firm, or soft. In a water CPS, the correctness of the system behavior depends not only on the logical results of the computations, but also on the physical instant at which these results are produced. A missed deadline in a water CPS for real-time water quality monitoring and pollution detection is catastrophic, and a missed deadline in a water CPS for the control and mitigation of water losses can lead to a significant loss. Hence the predictability of the system behavior is the most important concern in a water CPS. The predictability is often achieved by either static or dynamic scheduling of realtime tasks to meet their deadlines. Static scheduling makes scheduling decisions at compile time and is off-line. Dynamic scheduling is online and uses schedulability test to determine whether a set of tasks can meet their deadlines.

\section{CONCLUSIONS}

The world is facing severe challenges related to water sustainability. At the same time, information and communications technologies are rapidly advancing and are expected to capture and analyze data at a scale without precedent. With the potential to make remote sensing of the aquatic environment ubiquitous, cyber-physical systems can improve decision making with respect to many threats to water security, including the challenges discussed herein (persistent water quality problems and emergency contamination events) and a host of others (e.g., availability and use of surface and groundwater, flood forecasting and response, and prediction of climate change impacts).

Real-time and in-situ data acquisition for better understanding of water sustainability challenges and identification of effective solutions requires crosscutting research. Given the pressing needs and the depth and complexity of the challenges, researchers must interact with water sector professionals to support improved decision making with the best-available science and technology, and water management agencies must continuously adapt their strategies as better tools become available.

\section{REFERENCES}

[1] L. I. Millett and D. L. Estrin, Computing Research for Sustainability. Washington, DC: The National Academies Press, 2012.

[2] J. Hall, A. D. Zaffiro, R. B. Marx, P. C. Kefauver, E. R. Krishnan, R. C. Haught, and J. G. Herrmann, "On-line water quality parameters as indicators of distribution system contamination," J. Amer. Water Works Assoc., vol. 99, no. 1, pp. 66-77, 2007.

[3] M. V. Storey, B. van der Gaag, , and B. P. Burns, "Advances in on-line drinking water quality monitoring and early warning systems," Water Research, vol. 45, pp. 741-747, 2011.

[4] A. Jang, Z. Zou, K. K. Lee, C. H. Ahn, and P. L. Bishop, "State-ofthe-art lab chip sensors for environmental water monitoring," Meas. Sci. Technol., 2011.

[5] J. P. Bearinger, S. Terrettaz, and R. Michel, "Sulfamer block copolymers protection of gold surfaces," Nature Materials, no. 2, p. 257264, 2003.

[6] C. H. Choi, A. J. Scardino, P. G. Dylejko, L. E. Fletcher, and R. Juniper, "The effect of vibration frequency and amplitude on biofouling deterrence," Biofouling, vol. 29, no. 2, pp. 195-202, 2013.

[7] T. A. Nguyen, Y. Lu, X. Yang, and X. Shi, "Carbon and steel surfaces modified by Leptothrix discophora SP-6: Characterization and implications," Environmental science \& technology, vol. 41, no. 23, pp. 79877996, 2007.

[8] D. Pompili, T. Melodia, and I. F. Akyildiz, "Deployment analysis in underwater acoustic wireless sensor networks," in Proc. of the ACM Intl. Workshop on Underwater Networks (WUWNet), Los Angeles, CA, USA, 2006.

[9] H. Y. Schive, U. H. Zhang, and T. Chiueh, "Directionally unsplit hydrodynamic schemes with hybrid MPI/OpenMP/GPU parallelization in AMR," International Journal of High Performance Computing Applications, vol. 26, no. 4, pp. 367-377, 2012.

[10] Z. Shang, "Impact of mesh partitioning methods in CFD for large scale parallel computing," Computers \& Fluids, vol. 103, no. 1, pp. 1-5, 2014. 
[11] C. Chen, H. Liu, and R. C. Beardsley, "An unstructured grid, finitevolume, three-dimensional, primitive equations ocean model: Application to coastal ocean and estuaries," J. Atmos. Oceanic Technol., vol. 20, pp. 159-186, 2003.

[12] E. Brill, Securing Water and Wastewater Systems. Springer International Publishing, 2014, ch. 4: Implementing Machine Learning Algorithms for Water Quality Event Detection: Theory and Practice, pp. 107-122.

[13] S. E. Haupt, G. S. Young, and C. T. Allen, "A genetic algorithm method to assimilate sensor data for a toxic contaminant release," Journal of Computers, vol. 2, no. 6, pp. 85-93, 2007.

[14] E. Towler, M. Roberts, B. Rajagopalan, and R. S. Sojda, "Incorporating probabilistic seasonal climate forecasts into river management using a risk-based framework," Water Resources Research, vol. 49, no. 8, pp. 4997-5008, 2013.

[15] E. M. Zechman, "Agent-based modeling to simulate contamination events and evaluate threat management strategies in water distribution systems," Risk Analysis, vol. 31, p. 758772, 2011.

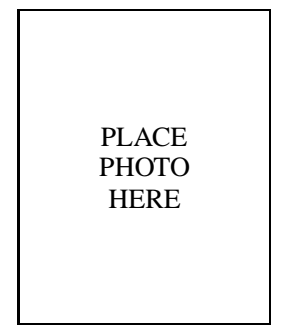

Zhaohui Wang (S'10-M'13) received a B.S. degree in 2006 from the Beijing University of Chemical Technology (BUCT), an M.S. degree in 2009 from the Institute of Acoustics, Chinese Academy of Sciences (IACAS), Beijing, China, and a Ph.D. degree from the University of Connecticut (UCONN), Storrs, all in electrical engineering. Dr. Wang has been with the Department of Electrical and Computer Engineering at the Michigan Technological University (Michigan Tech), Houghton, as an assistant professor since 2013. Her research interests lie in the areas of wireless communications, networking, and statistical signal processing, with recent focus on wireless communications and networking in underwater acoustic environments.

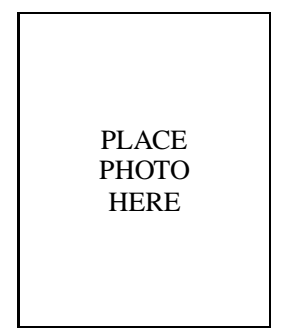

Houbing Song (M'12-SM'14) received the Ph.D. degree in electrical engineering from the University of Virginia, Charlottesville, VA, in 2012, and the M.S. degree in civil engineering from the University of Texas, El Paso, TX, in 2006. In 2012, he joined the Department of Electrical and Computer Engineering, West Virginia University, Montgomery, WV, where he is currently an Assistant Professor and the founding director of both the West Virginia Center of Excellence for Cyber-Physical Systems (WVCECPS) sponsored by the West Virginia Higher Education Policy Commission and the Security and Optimization for Networked Globe Laboratory (SONG Lab). He worked with Texas A\&M Transportation Institute (TTI), Texas A\&M University System, as an Engineering Research Associate in 2007. His research interests lie in the areas of cyberphysical systems, internet of things, intelligent transportation systems, wireless communications and networking, and optical communications and networking. Dr. Song's research has been supported by the West Virginia Higher Education Policy Commission.

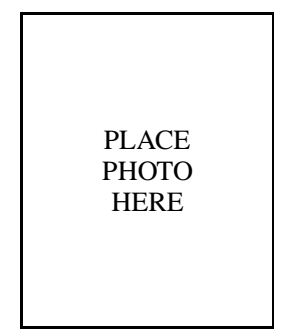

David Watkins received B.S. degrees in civil engineering and engineering \& public policy from Washington University in St. Louis in 1991. He received an M.S. degree in environmental health engineering in 1992 and a Ph.D. in civil engineering in 1997, both from the University of Texas at Austin. Following two years as a research hydraulic engineer for the U.S. Army Corps of Engineers, Dr. Watkins joined the faculty of Civil and Environmental Engineering at Michigan Technological University in Houghton, Michigan, where he has been employed since 1999. His research focuses on modeling and analysis to support water resources sustainability, with recent projects addressing water scarcity in Florida and Texas and water pollution in the Great Lakes.

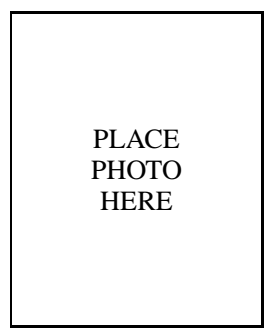

Keat Ghee Ong received his B.S., M.S., and $\mathrm{Ph} . \mathrm{D}$. degrees in Electrical Engineering from the University of Kentucky in 1997, 1998, and 2000, respectively. Currently, he is an associate professor in the department of Biomedical Engineering and director of Biotechnology Research Center at Michigan Technological University, Houghton, MI. His areas of expertise include wireless sensor networks, embedded sensors, implantable biosensors and biological sensing materials and devices. He is also working on magnetism, RF, and acoustic-based sensors, magnetoelastic materials, and measurement technique and instrument automation. Ong is the Associate Editor of Sensor Letters, and has published over 100 publications in archival journals and conference proceedings.

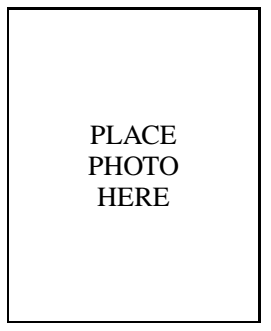

Pengfei Xue received his B.S.(2000) in applied mathematics from the East China Normal University and his Ph.D. (2012) in Marine and Atmospheric System Modeling and Analysis (MASMA) from University of Massachusetts. Dr. Xue is an assistant professor in the Department of Civil and Environmental Engineering at the Michigan Technological University (Michigan Tech). Prior to joining Michigan Tech in 2013, He worked as a postdoc associate in the department of Earth, Atmospheric and Planetary Sciences (EAPS), Massachusetts Institute of Technology (MIT) since 2012. Dr. Xue's research focus on the development and application of numerical models to problems in the coastal and regional oceans and the Great Lakes. His research interests include hydrodynamic modeling, dynamics of coupled ocean/lake-atmosphere models, ocean data assimilation, estuary and coastal ocean circulation.

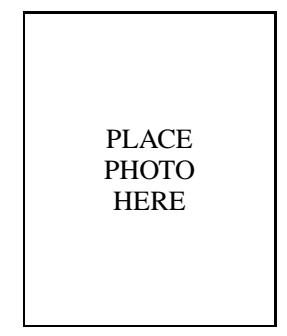

Qing Yang (M'11), Ph.D, is an RightNow Technologies Assistant Professor in the Department of Computer Science, Montana State University (MSU). He received B.S. and M.S. degrees in Computer Science from Nankai University and Harbin Institute of Technology, China, in 2003 and 2005, respectively. He received Ph.D degree in Computer Science from Auburn University in 2011. His research interests lie in the areas of wireless sensor network and trust assessment in online social networks. He serves as a TPC for several international conferences and on the editorial boards of several journals. His research has been funded by FHWA and DOT.

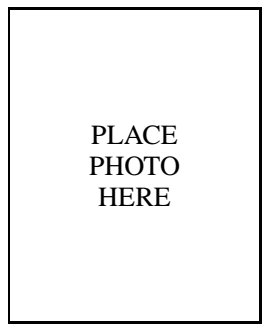

Xianming Shi received a B.S. degree in 1993 from Beijing Institute of Chemical Technology and M.S. degrees in 1996 from Tianjin University and in 2002 from Montana State University, respectively. $\mathrm{He}$ received his Ph.D. degree from the Institute of Chemistry, Chinese Academy of Sciences, Beijing, China in 1999. Dr. Shi is an Associate Professor in Civil \& Environmental Engineering at the Washington State University. During 2004 to 2014, he was a Program Manager and Senior Research Scientist at the Western Transportation Institute and a research faculty at the Montana State University. His research interests include smart and green infrastructure technologies, bio-energy harvesting, and sustainable materials. 